\title{
Restauraciones estéticas implanto soportadas: principios biológicos y clínicos
}

\section{Aesthetic implant supported restorations: biologic and clinical principles}

\author{
ARDILA MEDINA CIM*
}

Ardila Medina CM. Restauraciones estéticas implanto soportadas: principios biológicos y clínicos. Av Periodon Implantol. 201 1; 23, 2: 129-135.

\begin{abstract}
RESUMMN
El nivel del soporte óseo y las dimensiones de los tejidos blandos alrededor de las restauraciones implanto soportadas (RISs) son factores fundamentales para conseguir un resultado estético óptimo en el tratamiento con implantes. Otros elementos que también influyen en la remodelación ósea después de la colocación de implantes tienen que ver con su localización con relación a los dientes vecinos así como su vínculo a la dimensión buco lingual del reborde alveolar. La pérdida ósea puede influir negativamente en la disposición de los tejidos blandos, y a su vez el espesor de la mucosa del aspecto bucal del implante puede también mediar sobre la altura de los tejidos blandos supracrestales y en el resultado estético de la restauración con implantes. Las dimensiones de la corona, la posición del punto de contacto, la distancia implante diente y el diámetro del implante son componentes adicionales que intervienen en la configuración de los tejidos blandos, en la estética y funcionalidad de las RISs.
\end{abstract}

PALABRAS CLAVE: Implantes dentales, tejidos blandos, estética.

\section{SUMMARY}

The level of bone support and the soft tissue dimensions around the implant supported restoration are factors suggested to be important for the esthetic outcome of implant therapy. Implant position in relation to neighboring teeth as well as in relation to the buccal lingual dimension of the alveolar ridge are factors shown to influence the degree of bone remodeling following implant placement. Bone loss may negatively influence the soft tissue topography and the esthetic outcome of the implant therapy, beside, the thickness of the mucosa at the facial aspect of the implant may also influence the height of the supra crestal soft tissue portion. Other factors indicated to influence the position of the soft tissues topography and esthetics at implant-supported restorations are crown dimensions, contact point position, tooth-implant distances and implant diameter.

KEY WORDS: Dental implants, soft tissues, esthetics.

Fecha de recepción: 2 de marzo 2009.

Fecha de aceptación: 7 de marzo 2009.

\section{INTRODUCCIÓN}

Es fundamental obtener un equilibrio armonioso entre los aspectos biológicos, funcionales y estéticos para que las restauraciones implanto soportadas (RISs) sean consideradas exitosas. Lo anterior implica el reemplazo de los tejidos duros y un correcto perfil de los tejidos blandos, de tal manera que el implante pueda colocarse en la posición deseada como lo determina la restauración $(1,2)$. Debido a que el implante reempla- 
za la raíz del diente perdido y con el fin de establecer un perfil de emergencia estético, debe prestarse atención especial a la transición entre el tamaño apropiado del implante y la corona (1). Para obtener una estética natural de los tejidos blandos, el contorno, la altura y la amplitud gingival en el sitio del implante debe corresponder con la de los tejidos que rodean el diente adyacente. Debe existir una adecuada cantidad de hueso para colocar el implante, acompañado de un marco proporcionado de los tejidos blandos que incluya la papila interproximal y una zona apropiada de encía insertada (3). La ubicación estética de un implante se maneja teniendo presente principios biológicos y restauradores. Estéticamente el implante debe localizarse para satisfacer los parámetros de los contornos de tal forma que la restauración sea agradable. Biológicamente debe posicionarse para permitir el mantenimiento de los tejidos blandos y duros. El objetivo de este artículo es presentar los principios biológicos y clínicos protésico periodontales esenciales para incrementar la probabilidad de éxito de las RISs.

\section{PRINCIPIOS PERIODONTALES}

Es fundamental el conocimiento de la biología y la anatomía del periodonto para la manipulación de los tejidos blandos alrededor de los implantes. Los principios periodontales a tener en cuenta en una RIS incluyen aspectos relacionados con el complejo periimplantar (PIM), la anatomía ósea y los tejidos mucogingivales.

Es imprescindible tener en cuenta que la anatomía del reborde alveolar incluye los tejidos blandos y el soporte óseo en todas sus dimensiones, y que el contorno de los tejidos blandos alrededor del implante están fuertemente afectados por la anatomía ósea. Los complejos dentogingival y PIM son similares en su composición celular, constituidos por epitelio oral queratinizado y epitelio de unión no queratinizado (4). Histológicamente, estos tejidos difieren en la forma como se adhieren al diente y a los implantes. Mientras que en la dentición natural el epitelio de unión se inserta mediante glucoproteínas, en los implantes se lleva a cabo una seudo-inserción mediante hemidesmosomas (5). Además, las fibras de tejido conectivo se insertan mecánicamente en el cemento radicular, particularidad que no se observa en los implantes (6). A cambio, se percibe un tejido conectivo fuertemente adherido alrededor del titanio (6). Numerosos estudios experimentales han revelado que el concepto de amplitud biológica (4), fundamental para determinar la estéti- ca, puede también ser aplicado a los implantes oseointegrados, debido a que los tejidos blandos también muestran dimensiones relativamente constantes alrededor de los implantes, aún después de la carga protésica (3).

Para conseguir una RIS exitosa, el hueso que rodea el implante debe tener una configuración tridimensional que permita la ubicación de un implante en una posición restauradora ideal (1). Diferentes ayudas como la evaluación clínica detallada, mapeo, tomografías y encerado diagnóstico permiten visualizar las deficiencias tisulares y la posición final del diente. Son fundamentales dos estructuras anatómicas para determinar la predictibilidad de los tejidos blandos después de la ubicación de los implantes: la altura y espesor de la cresta ósea vestibular y la altura de la cresta alveolar interproximal (1). Kois y Kan (7), evaluaron 100 pacientes clasificados según la distancia vertical encontrada desde la cresta ósea al margen gingival. Mientras esta distancia fuera mayor la posibilidad de pérdida de tejido después de un procedimiento invasivo era más alta.

La pérdida de la cresta ósea que ocurre alrededor de los implantes tiene un componente horizontal y vertical. Después de la conexión pilar-implante, la cresta ósea se reabsorbe horizontalmente 1,3-1,4 mm (9). La pérdida ósea horizontal, puede influir sobre la altura ósea interproximal si los implantes no se colocan lo suficientemente lejos del diente adyacente $(1,5 \mathrm{~mm}) \circ$ de otros implantes ( $3 \mathrm{~mm}$ ) (8), incrementando la distancia al punto de contacto, influyendo sobre la estética. Por otra parte, la pérdida ósea vertical es una situación clínica bastante compleja, debido que no se cuenta con técnicas predecibles para aumentar la altura de la cresta ósea. Cuando se cuenta con una pared ósea de suficiente altura y espesor es importante estabilizar a largo plazo los márgenes gingivales alrededor de implantes y dientes adyacentes (3). La colocación de implantes en sitios con defectos óseos bucales necesariamente estarán asociados a recesión gingival y exposición de los pilares de los implantes. Se han propuesto varias técnicas quirúrgicas para corregir tales defectos óseos incluyendo injertos óseos (10), regeneración ósea guiada usando membranas (11), combinación de bloques óseos y membranas (12), y distracción osteogénica (13). Estudios experimentales han demostrado que el aumento óseo horizontal es predecible con técnicas de regeneración (11), mientras que la ganancia vertical de hueso es un procedimiento en donde es más difícil obtener resultados exitosos (14). 
La necesidad de mantener encía insertada y queratinizada alrededor de los implantes puede ser objeto de debate, pero es muy evidente la importancia de estos tejidos alrededor de los márgenes de la restauración. Específicamente, la encía insertada es fundamental para reducir la probabilidad de recesión gingival en áreas que requieran márgenes estéticos y también con el fin de facilitar las impresiones.

Los márgenes de algunas restauraciones se deben extender ligeramente en el surco gingival debido a que lo requieren las exigencias estéticas. Tal extensión debe considerarse como un compromiso biológico que debe realizarse con precaución (15). Para disminuir la posibilidad de recesión, los tejidos gingivales deben estar clínicamente sanos antes de iniciar los procedimientos restauradores presentando además una adecuada banda de encía insertada y queratinizada (16).

La exactitud de las impresiones definitivas subgingivales depende de la exposición adecuada de los márgenes de la preparación dentaria, procedimiento que se logra con mayor éxito cuando los tejidos blandos son retraídos atraumáticamente en presencia de encía insertada y queratinizada (15).

Otro aspecto importante que debe tenerse en cuenta en la RIS es el biotipo periodontal. Se han descrito dos biotipos periodontales diferentes relacionados con la morfología interdental y la arquitectura ósea: periodonto delgado y grueso (17). El biotipo delgado se encuentra en menos del $15 \%$ de los casos y se caracteriza por poseer un tejido blando delicado, festoneado del hueso subyacente, presencia de dehiscencias y fenestraciones, y una cantidad reducida de encía queratinizada (1). Además, por lo general la papila interproximal no llena el espacio entre los dientes vecinos (18). Las particularidades del biotipo delgado juegan un papel importante en la ubicación de la terminación del pilar del implante, la cual debe posicionarse ligeramente hacia lingual, si el pilar empleado es de titanio, con el fin de enmascarar su traslucidez. En estos casos se debe tener precaución con la consecución de un perfil de emergencia óptimo (1). Otros aspectos importantes de los dientes de aquellos pacientes con periodonto delgado es que se caracterizan por tener una forma triangular y por presentar áreas de contacto reducidas en sentido bucolingual, coronalapical, y hueso interproximal muy delgado (17).

El periodonto grueso se identifica por poseer un tejido blando más fibrótico, hueso subyacente aplanado y adecuada cantidad de encía queratinizada. Las áreas de contacto son más amplias bucolingualmente y coronoapicalmente, estando localizadas más hacia el tercio medio del diente; las coronas anatómicas son generalmente cuadradas (18).

La forma de los dientes vecinos influye también en la forma de la RIS. La RIS debe copiar fielmente el diente contra lateral natural especialmente en su tercio coronal y hasta el margen gingival (1). Se debe desplegar un delicado equilibrio que proporcione un soporte adecuado a la arquitectura gingival sin generar excesiva presión. Idealmente el contorno vestibular debe ser levemente más plano que el diente natural para disminuir el desplazamiento apical del margen libre de la encía después de la instalación de la RIS (18).

\section{PRINCIPIOS PROTÉSICOS}

La posición en la cual se localiza el implante es de vital importancia, por esta razón la RIS debe ser racionalizada como una extensión de la corona clínica en el hueso alveolar (7). Esto es posible solamente si el implante se localiza correctamente en todas sus dimensiones: coronal-apical, mesial-distal y vestibular-lingual (3). Los principios protésicos deben tener presente consideraciones estéticas y aspectos relacionados con la restauración provisional, cementación y selección de pilares previos a la restauración definitiva.

Los principios que son a su vez prerrequisitos para conseguir RISs estéticas son: presencia de un adecuado volumen óseo para la colocación del implante (1), precisión en la ubicación del implante (3), estabilidad en la interfase implante-pilar (2), microespacio mínimo entre el pilar y el implante (19), adecuado perfil de emergencia (14) y apariencia similar al diente adyacente (15).

Las restauraciones temporales sirven como patrón para la cicatrización tisular siendo una herramienta diagnóstica para obtener buenos resultados protésicos. También permiten una evaluación de la restauración final en estados tempranos del tratamiento y admiten correcciones $o$ alteraciones previas a la cementación definitiva de la RIS. Algunas funciones de los provisionales son: mantener espacio y proteger el pilar mientras se realiza la restauración final, facilitar la cicatrización y la higiene oral, guiar los tejidos blandos con el fin de establecer contornos ideales, restablecer la dimensión vertical y el esquema oclusal, servir de anclaje para movimientos ortodóncicos, permitir evaluar 
la estética, la fonética, así como la forma y función, visualizando previamente el resultado final (3).

Se debe tener precaución para no dejar excesos de material cementante durante el procedimiento de cementación de la RIS ya que favorecen procesos inflamatorios gingivales. Los excesos pueden ser evitados si se aplican mínimas cantidades de cemento (20). Es de utilidad también, antes de la cementación, aplicar el cemento en la corona y colocarlo sobre un pilar análogo. De esta forma el exceso de cemento puede ser eliminado y posteriormente se lleva la corona al pilar en la cavidad bucal (19).

Los pilares empleados en las RISs pueden ser de dos tipos: pilares de cicatrización y pilares definitivos. Davidoff (21), estableció que la expansión de los tejidos blandos se realiza a través del uso de componentes de mayor diámetro, como los pilares de cicatrización, para crear un contorno adecuado de los tejidos blandos y crear un perfil de emergencia para la restauración definitiva. Sin embargo, Askary (22), sugiere que pilares de cicatrización cortos y delgados ayudan a preservar la integridad de los tejidos blandos, disminuyendo el riesgo de recesión y reduciendo la posibilidad de daño sobre estructuras vitales.

La selección de los pilares y restauraciones definitivas son un aspecto de preferencia personal. Los pilares disponibles incluyen los de tipo atornillados, individualizados y cerámicos y las restauraciones definitivas pueden ser cementadas y atornilladas. Las restauraciones cementadas ofrecen superioridad en oclusión, estética, pasividad y características de carga cuando se comparan con las atornilladas (23). Sin embargo, una investigación realizada por Cicciù y colaboradores (24), informa un éxito del 100\% en 84 RISs atornilladas, evaluadas durante 44,3 meses de seguimiento. Los pilares individualizados tienen contornos y líneas de terminación que pueden ser alterados para que coincidan con las dimensiones tisulares (25). Los pilares cerámicos tienen las ventajas de otros pilares más la dimensión del color. Pueden ser usados especialmente en pacientes con periodonto delgado y de esa manera evitar la traslucidez producida por pilares metálicos (25).

\section{CONSIDERACIONES OCLUSALES}

Las fuerzas masticatorias desplegadas por un paciente restaurado con una RIS son equivalentes a aquellas de una dentición natural (26). Sin embargo, existen estudios que demuestran que los implantes son más sus- ceptibles a sobrecarga oclusal (27). Durante el plan de tratamiento se debe realizar una evaluación general de la probabilidad de carga que va a ser soportada por la RIS (28). Algunos aspectos como el volumen y densidad ósea, cargas tempranas, y el diseño de la restauración deben revisarse antes de determinar el número, longitud y diámetro de los implantes. Los implantes están anquilosados alrededor del hueso sin intervención del ligamento periodontal y su rango promedio de movimiento es de aproximadamente 3-5 micrones $(29,30)$.

Se deben emplear técnicas para disminuir las cargas excesivas sobre la RIS. Se recomienda una guía anterior y una desoclusión posterior en los movimientos de lateralidad. Se acepta una función de grupo si el canino está comprometido y preferiblemente el contacto oclusal inicial debe presentarse en la dentición natural (28).

Un punto de controversia planteado en la literatura es el papel de la oclusión en el fracaso de los implantes sin fractura. Los posibles agentes responsables de la pérdida ósea de los implantes que han fallado son: fuerzas traumáticas oclusales, ajuste inadecuado de la restauración, inflamación inducida por bacterias o una combinación de estos factores (26).

La ferulización de los dientes puede aumentar la comodidad de los pacientes y mejorar la función masticatoria (3). En algunos casos avanzados, la ferulización puede estar indicada tempranamente con el fin de mejorar la función, realzar la estética o permitir la colocación de implantes bajo restauraciones provisionales (3). Los implantes pueden ser ferulados para proporcionar estabilidad adicional a la prótesis e incrementar el soporte. Las contraindicaciones de la ferulización de implantes incluyen un reducido acceso a la higiene bucal y un posible compromiso estético (3).

\section{ESTABILIDAD DE LOS TEJIDOS BLANDOSY DE LOS CONTORNOS ALREDEDOR DE LAS RESTAURACIONES IMPLANTO SOPORTADAS}

La estabilidad de los tejidos blandos (ETB) alrededor de RISs y dientes adyacentes es muy importante desde el punto de vista estético. Scheller y colaboradores (31), evaluaron en un estudio multicéntrico prospectivo la ETB en 99 RISs. La tasa acumulada de éxito fue del $96 \%$ para los implantes y del $91 \%$ para las coronas. Durante los cinco años de seguimiento, permaneció estable el ETB alrededor de las RISs y de los dientes adyacentes. Se realizó un ensayo clínico 
aleatorizado en 55 sujetos con 63 RISs con el fin de evaluar el contorno gingival restaurado por medio de provisionales durante dos años (32). Los resultados indicaron que el uso de restauraciones provisionales puede restituir el contorno de los tejidos blandos más rápido que los pilares de cicatrización solos, sin embargo, la papila adyacente a la RIS presentó similar volumen en los dos grupos. En un estudio se evaluó la estabilidad de la mucosa alrededor de 10 RISs superiores y sus dientes adyacentes siguiendo el mismo protocolo, el cual incluyó regeneración ósea guiada e injerto de tejido conectivo (33). Un año después de la instalación de las RISs el tejido blando de su superficie bucal se contrajo $0.6 \mathrm{~mm}$ en promedio. Sin embargo, el volumen del tejido blando en el área de la papila incrementó $0,4 \mathrm{~mm}$ en promedio y ninguna de ellas perdió espesor. Giannopoulou y colaboradores (34), estudiaron el efecto de márgenes intracreviculares sobre la salud periimplantar (PIM) en 61 RISs. Los sujetos fueron aleatoriamente seleccionados y examinados. Se registraron parámetros clínicos, microbiológicos y biomecánicos a nivel base y después de tres hasta nueve años. La única diferencia estadísticamente significativa entre los dos tiempos de evaluación estuvo relacionada con un aumento leve tanto en la profundidad al sondaje (nivel base 4,2 $\pm 1,4 \mathrm{~mm}$, después de tres años 4,6 $\pm 1,4 \mathrm{~mm} ; \mathrm{p}=0,001$ ), como en la distancia entre el hombro del implante y el margen de la mucosa (nivel base $-3,3 \pm 1,5 \mathrm{~mm}$, después de tres años $3,6 \pm 1,4 \mathrm{~mm} ; \mathrm{p}=0,001)$. Los autores concluyeron que en pacientes con una higiene bucal apropiada, la posición intracrevicular del margen de las RISs no afectó adversamente la salud y estabilidad PIM. Hall y colaboradores (35), mediante un ensayo clínico controlado, evaluaron después de un año los resultados restauradores y estéticos de la mucosa PIM al comparar implantes restaurados inmediatamente $(n=14)$ e implantes restaurados convencionalmente en dos fases $(n=14)$. No se encontraron diferencias estadísticamente significativas entre los dos grupos de comparación en aspectos relacionados con la pérdida ósea, la ETB y el mantenimiento protésico. Se realizó un estudio retrospectivo en 45 pacientes llamados para evaluación de sus RISs después de 2-4 años de haber sido instaladas (36). Los implantes mostraron estabilidad sin signos de infección PIM; los tejidos estaban clínicamente saludables y no se observó recesión del margen de la mucosa vestibular. Se evaluó radiográfica y clínicamente el hueso PIM y las condiciones de la mucosa de 43 implantes en 39 sujetos (37). Este estudio prospectivo determinó la tasa de éxito, las complicaciones protésicas y la respuesta de los tejidos PIM asociados con carga funcional posterior a tres semanas y la coloca- ción de implantes de una sola fase, evaluados después de tres años de instaladas las RISs. Tres de los implantes fallaron en el primer año. El cambio en el nivel del hueso marginal fue de $0,42 \pm 0,59 \mathrm{~mm}$ después del tercer año. La papila se midió en el primer y tercer año $(0,61 \pm 0,95 \mathrm{~mm}$ y $0,74 \pm 0,79 \mathrm{~mm}$, respectivamente). Las dimensiones bucales de los tejidos PIM a nivel del cénit gingival también incrementaron en el mismo período (primer año: $0,34 \pm 0,94 \mathrm{~mm}$; tercer año: $0,51 \pm 1,42$ $\mathrm{mm}$ ). Schrop y colaboradores (38), realizaron una investigación en la que evaluaron después de cinco años de seguimiento los resultados restauradores y tisulares de implantes sometidos a carga temprana y tardía. Antes de la carga oclusal fracasaron dos implantes del grupo de carga temprana y un implante del grupo de carga tardía. La apariencia de los tejidos blandos fue levemente mejor en el grupo de carga temprana inmediatamente después de la instalación de la restauración, sin embargo, mejoró espontáneamente sobre el tiempo y no se encontró diferencia estadísticamente significativa entre los dos protocolos después de cinco años.

Como se presentó anteriormente, la gran mayoría de estudios muestran resultados positivos cuando evalúan la ETB alrededor de las RISs, sin embargo, algunos investigadores presentan conclusiones menos alentadoras. Chang y colaboradores (39), compararon las coronas y la ETB entre 41 RISs y dientes naturales contralaterales, en 29 pacientes evaluados durante al menos seis meses. Se observó, que las coronas de los implantes presentaron una mayor longitud y una menor amplitud bucolingual, estaban rodeadas de una mucosa vestibular gruesa y mostraron alta frecuencia de mucositis, sangrado al sondaje, mayor profundidad al sondaje y una menor altura de la papila distal. Por su parte, Cardaropoli y colaboradores (40), realizaron un estudio prospectivo con el fin de evaluar clínica y radiográficamente las alteraciones dimensionales de los tejidos PIM en RISs en el momento de la instalación del implante y un año después de cargado. Once pacientes entre 18 y 36 años fueron incluidos en el análisis. El procedimiento se realizó en dos etapas con un intervalo de cicatrización de seis meses. Radiográficamente se observó una pérdida ósea vestibular y lingual de 0,7 mm a 1,3 mm en el momento de la conexión del pilar $(p<0,05)$. Se incrementó el espesor de la mucosa labial en el momento de la instalación de la corona seguido por una remisión leve después de un año. Durante el intervalo correspondiente, se observó un desplazamiento apical del margen gingival $(0,6 \mathrm{~mm}$; $\mathrm{p}<0,05)$. Los autores concluyeron que las alteraciones observadas después de la ubicación de la corona pueden afectar la estética de una RIS. 


\section{CONCLUSIONES}

La aplicación de los principios biológicos y clínicos anteriormente descritos son fundamentales para el éxito protésico periodontal de una RIS. En áreas de interés estético una adecuada banda de encía insertada puede favorecer la comodidad del paciente, reducir la posibilidad de recesión gingival y simplificar los procedimientos restauradores. Preferiblemente, en un surco gingival saludable, los márgenes restauradores no deben ser ubicados a una profundidad mayor a $0,5 \mathrm{~mm}$. Los implantes funcionan mas adecuadamente cuando las fuerzas oclusales están dirigidas verticalmente y se sugiere que los dientes naturales guíen la oclusión en pacientes parcialmente edéntulos. Las restauraciones provisionales son una parte integral de la terapia periodontal y restauradora en las RISs, debido a que pueden ser usadas para establecer contornos estéticos y fisiológicos que pueden ser mantenidos saludablemente por los pacientes.

\section{BIBLIOGRAFÍA}

1. Jivraj S, Chee W. Treatment planning of implants in the aesthetic zone. Br Dent J 2006;201:77-89.

2. Velasco Ortega E, Fornés Ortuño E, García Méndez A, Medel Soteras R, López Frías J. La carga inmediata con implantes Microdent en el maxilar superior: I. Aspectos quirúrgicos. Av Periodon Implantol 2007;19 Suppl:9-16.

3. Yeung SC. Biological basis for soft tissue management in implant dentistry. Aust Dent J 2008;53 Suppl 1:S39-42.

4. Padbury A Jr, Eber R, Wang HL. Interactions between the gingiva and the margin of restorations. J Clin Periodontol 2003;30:379-85.

5. Linkevicius T, Apse P. Influence of abutment material on stability of peri-implant tissues: a systematic review. Int J Oral Maxillofac Implants 2008; 23:449-56.

6. Hermann F, Lerner H, Palti A. Factors influencing the preservation of the periimplant marginal bone. Implant Dent 2007;16:165-75.

7. Kois J C, Kan J Y. Predictable per-implant gingival aaesthetics. Surgical and prosthodontic rationales. Prac Perio Aest Dent 2001;13:691-8.

8. Tarnow DP, Cho SC, Wallace SS. The effect of interimplant distance on the height of inter-implant bone crest. J Periodontol 2000;71:546-9.
9. Degidi M,Novaes AB Jr,Nardi D, Piattelli A. Outcome analysis of immediately placed, immediately restored implants in the esthetic area: the clinical relevance of different interimplant distances. J Periodontol 2008;79: 1056-61.

10. Cordaro L, Amade DS, Cordaro M. Clinical results of alveolar ridge augmentation with mandibular block bone grafts in partially edentulous patients prior to implant placement. Clin Oral Implants Res 2002:13:103-11.

11. Wang HL, Carrol MJ. Guided bone regeneration with bone grafts and collagen membranes. Quintessence Int 2001;32:504-15.

12. Buser D, Dulak K, Hirt HP, Schenk RK. Lateral ridge augmentation using autografts and barrier membranes. A clinical study in 40 partially edentulous patients.J Oral Maxillofac Surg 1996; 54:420-32.

13. Rachmiel A, Srouji S, Peled M. Alveolar ridge augmentation by distraction osteogenesis. Int J Oral Maxillofac Surg 2001;30:510-17.

14. Simion M, Jovanovic SA, Tinti C, Benfenati SP. Long term evaluation of osseointegraded implants inserted at the time or after vertical ridge augmentation. A retrospective study on 123 implants with 1-5 years following-up. Clin Oral Implants Res 2001;12:35-45.

15. Gómez Mira F, Ardila Medina CM. Contornos y perfil de emergencia: aplicación clínica e importancia en la terapia restauradora. Av. Odontoestomatol 2009;25:171-9.

16. Flanagan D. Fixed partial dentures and crowns supported by very small diameter dental implants in compromised sites. Implant Dent 2008;17:182-91.

17. Becker W, Ochsenbein C, Tibbetts, Becker BE. Alveolar bone anatomic profiles as measured from dry skulls. Clinical ramifications. J Clin Periodontol 1997;24:727-31.

18. McGarry TJ. Identifying the aesthetic and functional determinants in the collapsed dentition. Dent Today 2008;27:108-11.

19. Hermann JS, Buser D, Schenk RK, Cochran DL. Crestal bone changes around titanium implants. A histometric evaluation of unloaded non-submerged and submerged implants in the canine mandible. J Periodontol 2000;71: 1412-24.

20. Pegoraro TA, da Silva NR, Carvalho RM. Cements for use in esthetic dentistry. Dent Clin North Am 2007;51: 453-71. 
21. Davidoff D. Developing soft tissue contours for implant supported restorations: A simplified method for enhanced aesthetics. Pract Periodontics Aesthet Dent 1996;8:507-13.

22. el Askary AS. Multifaceted aspects of implant esthetics: the anterior maxilla. Implant Dent 2001;10:182-91.

23. Michalakis KX, Hirayama H, Garefis PD. Cementretained versus screw-retained implant restorations: a critical review. Int J Oral Maxillofac Implants 2003;18: 719-28.

24. Cicciù M, Beretta M, Risitano G, Maiorana C. Cementedretained vs screw-retained implant restorations: an investigation on 1939 dental implants. Minerva Stomatol 2008;57:167-79.

25. Christensen GJ. Selecting the best abutment for a single implant. J Am Dent Assoc 2008;139:484-7.

26. Rilo B, da Silva JL, Mora MJ, Santana U. Guidelines for occlusion strategy in implant-borne prostheses. A review. Int Dent J 2008;58:139-45.

27. Miyata T, Kobayashi Y, Araki H, Ohto T, Shin K. The influence of controlled oclusal overload on peri-implant tissue. Part 3: a histologic study in monkeys. Int J Oral Maxillofac Implants 2000;15:425-31.

28. Jivraj S, CheeW. Treatment planning of implants in posterior quadrants. Br Dent J 2006;201:13-23.

29. Schulte W. Implants and the periodontium. Int Dent J 1995;45:16-26.

30. Cano-Sánchez J, Campo-Trapero J, Restoy-Lozano A, Bascones-Martínez A. Mecanobiología de los huesos maxilares: III. Regeneración ósea. Av Odontoestomatol 2008;24:227-237.

31. Scheller H, Urgell JP, Kultje C, Klineberg I, Goldberg PV, Stevenson-Moore P, et al. A 5-year multicenter study on implant-supported single crown restorations. Int J Oral Maxillofac Implants 1998;13:212-8.

32. Jemt T. Restoring the gingival contour by means of provisional resin crowns after single-implant treatment. Int J Periodontics Restorative Dent 1999;19:20-9.
33. Grunder U. Stability of the mucosal topography around single-tooth implants and adjacent teeth: 1-year results. Int J Periodontics Restorative Dent 2000;20:11-7.

34. Giannopoulou C, Bernard JP, Buser D, Carrel A, Belser UC. Effect of intracrevicular restoration margins on periimplant health: clinical, biochemical, and microbiologic findings around esthetic implants up to 9 years. Int J Oral Maxillofac Implants 2003;18:173-81.

35. Hall JA, Payne AG, Purton DG, Torr B, Duncan WJ, De Silva RK. Immediately restored, single-tapered implants in the anterior maxilla: prosthodontic and aesthetic outcomes after 1 year. Clin Implant Dent Relat Res 2007; 9:34-45.

36. Buser D, Bornstein MM, Weber HP, Grütter L, Schmid B, Belser UC. Early implant placement with simultaneous guided bone regeneration following single-tooth extraction in the esthetic zone: a cross-sectional, retrospective study in 45 subjects with a 2- to 4-year follow-up. J Periodontol 2008;79:1773-81.

37. Cooper LF, Ellner S, Moriarty J, Felton DA, Paquette D, Molina A, et al. Three-year evaluation of single-tooth implants restored 3 weeks after 1-stage surgery. Int J Oral Maxillofac Implants 2007;22:791-800.

38. Schrop L, Isidor F. Clinical outcome and patient satisfaction following full-flap elevation for early and delayed placement of single-tooth implants: a 5-year randomized study. Int J Oral Maxillofac Implants 2008;23:733-43.

39. Chang M, Odman PA, Wennström JL, Andersson B. Esthetic outcome of implant-supported single-tooth replacements assessed by the patient and by prosthodontists. Int J Prosthodont 1999;12:335-41.

40. Cardaropoli G, Lekholm U,Wennström JL.Tissue alterations at implant-supported single-tooth replacements: a 1-year prospective clinical study. Clin Oral Implants Res 2006; 17:165-71.

\section{CORRESPONDENCIA}

Carlos Martín Ardila Medina

Carrera 47 No. 20 sur 46 Envigado Antioquia Colombia 57(4) 3348122

cmartin@odontologia.udea.edu.co 